\title{
Importancia de una propuesta para la implementación de un programa de tamizaje neonatal expandido en Colombia
}

Danik Valera Antequera ${ }^{\mathbf{a}}$ - Ana Lorena Montealegre Páez ${ }^{\mathbf{b}}$

- Antonio Bermúdezc - Reggie García Robles ${ }^{\mathbf{b}}$

\begin{abstract}
Resumen: El tamizaje neonatal expandido permite la detección temprana de diversos errores innatos del metabolismo. En Colombia, las condiciones para llevar adelante un programa nacional de alto impacto en salud pública están dadas. A través de una búsqueda bibliográfica sobre el tema en diferentes países, se realizó una disertación sobre la implementación de un programa nacional de tamizaje neonatal. Esto con el fin de plantear una propuesta de tamizaje neonatal expandido por espectrometría de masas en tándem en Colombia, completo, conciso, detallado y acorde con la legislación colombiana, las necesidades y las características de la población. Implementar un programa nacional de este tipo supone un gran impacto en la salud pública y debe ser liderado por el Estado, con la participación y apoyo de profesionales de salud, academia, asociaciones de pacientes e industria farmacéutica.
\end{abstract}

Palabras clave: tamizaje neonatal; errores innatos del metabolismo; salud pública; mortalidad infantil; enfermedades genéticas congénitas

Fecha de recepción: 23 de abril de 2019 Fecha de aprobación: 28 de julio de 2019

Cómo citar: Valera Antequera D, Montealegre Páez AL, Bermúdez A, García Robles R. Importancia de una propuesta para la implementación de un programa de tamizaje neonatal expandido en Colombia. Revista Med. 27(2): 23-35. Disponible en: https://revistas.unimilitar.edu.co/index.php/rmed/article/view/4195

a Facultad de Medicina, Universidad El Bosque, Bogotá D.C., Colombia.

b Instituto de Investigación en Nutrición, Genética y Metabolismo. Facultad de Medicina, Universidad El Bosque. Bogotá D.C., Colombia. Correo electrónico: garciareggie@unbosque.edu.co

c Instituto Nacional de Salud. Bogotá D.C., Colombia. 


\title{
Importance of a Proposal for Implementing an Expanded Neonatal Screening Program in Colombia
}

\begin{abstract}
Expanded neonatal screening allows early detection of various inborn errors of metabolism. In Colombia, the conditions to carry out a national program with a high impact on public health are in place. Through a review of the international literature on the subject, this reflection on the implementation of a national neonatal screening program brings forward a complete, concise, detailed proposal for tandem mass spectrometry-expanded neonatal screening in Colombia that conforms to the legislation and the needs and characteristics of the population. Implementing such a national program has a great impact on public health and must be led by the State, with the participation and support of health professionals, academia, patient associations, and the pharmaceutical industry.
\end{abstract}

Keywords: Neonatal screening; inborn errors of metabolism; public health; child mortality; congenital genetic diseases

\section{Importância de uma proposta para a implementação de um programa de triagem neonatal ampliada na colômbia}

\begin{abstract}
Resumo: A triagem neonatal ampliada permite que vários erros inatos do metabolismo sejam identificados precocemente. Na Colômbia, as condições para a realização de um programa nacional com alto impacto na saúde pública estão disponíveis. Por meio de uma pesquisa bibliográfica sobre o assunto em diferentes países, foi realizada uma dissertação sobre a implementação de um programa nacional de triagem neonatal. A fim de apresentar uma proposta de triagem neonatal ampliada por espectrometria de massas em tandem na Colômbia, completa, concisa, detalhada e de acordo com a legislação colombiana, as necessidades e as características da população. A implementação de um programa nacional desse tipo tem um grande impacto na saúde pública e deve ser liderada pelo Estado, com a participação e o apoio de profissionais da saúde, da academia, das associações de pacientes e da indústria farmacêutica.
\end{abstract}

Palavras-chave: triagem neonatal; erros inatos do metabolismo; saúde pública; mortalidade infantil; doenças genéticas congênitas 


\section{Introducción}

Las enfermedades raras o huérfanas son un problema importante en salud pública, debido a que, aunque su prevalencia suele ser baja, en conjunto afectan a un porcentaje alto de la población. Sumado a esto, las personas que padecen estas enfermedades suelen recibir una atención médica de baja calidad, debido al desconocimiento médico, que retarda el diagnóstico; la falta de tratamientos médicos oportunos e, incluso, al escaso acceso a los servicios de salud. En 2005, veinticinco países de la Unión Europea tenían cerca de 30 millones de personas afectadas por este tipo de enfermedades; mientras tanto, en América Latina tanto su prevalencia como su incidencia no se conocen aún a ciencia cierta (1).

Justamente, los errores innatos del metabolismo (ЕIM), que forman parte de ese tipo de enfermedades, son un grupo de enfermedades de origen genético en las cuales se produce una alteración bioquímica, por compromiso de una proteína involucrada en algún proceso metabólico $(2,3)$. En su mayoría, estas enfermedades tienen un patrón de herencia autosómico recesivo y están clasificadas en los siguientes grupos principales:

1. eIM con cuadro de intoxicación: incluye las aminoacidopatías, las acidurias orgánicas, defectos del ciclo de la urea y la galactosemia (GAL). Suelen presentarse con un cuadro clínico de encefalopatía aguda, con períodos asintomáticos $(2,3)$.

2. eim por déficit energético: defectos de la $\beta$-oxidación de los ácidos grasos, la gluconeogénesis y la glucogénesis, las acidosis lácticas congénitas y las enfermedades mitocondriales. Su síntoma más común es la hipotonía muscular $(2,3)$.

3. EIM de moléculas complejas: están las enfermedades peroxisomales, lisosomales y déficit del metabolismo de los neurotransmisores. Son frecuentes las megalias y la disfunción neurológica $(2,3)$.

Las causas genéticas, los síndromes cromosómicos y las malformaciones congénitas asociadas suelen determinar, especialmente, la discapacidad intelectual en los individuos que padecen estas enfermedades. Dicha discapacidad ocasiona limitaciones al individuo dentro de la sociedad, pues suelen tener una pobre adaptabilidad a ella (4). Por ello, es necesaria la detección temprana y el tratamiento oportuno de estas patologías, con lo cual se evita o mitiga la discapacidad y la morbimortalidad de las personas afectadas. Con este objetivo, entonces, surgen los programas de tamizaje neonatal, implementados desde la década 1960, los cuales han mejorado con la inclusión de la espectrometría de masas en tándem (Ms/Ms) y la inclusión de estudios o pruebas para más EIM (5).

En Colombia, la Resolución 412 de 2000 estableció el tamizaje neonatal obligatorio para hipotiroidismo congénito [HC] (6). Sin embargo, este programa se encuentra enmarcado por "criterios de viabilidad, factibilidad y sostenibilidad económica" (7) y, comparado con el ámbito internacional, es un programa de alcance limitado, pues deja por fuera otros EIM que podrían ser tamizados (8).

Según las últimas recomendaciones del American College of Medical Genetics y el Maternal and Child Health Bureau, un programa de tamización expandido debe incluir 29 EIM, considerados objetivos principales, junto con 25 enfermedades clínicamente significativas, consideradas objetivos secundarios que pueden detectarse, adicionalmente, como diagnóstico diferencial de los desórdenes principales $(9,10)$.

Para la elaboración de una propuesta de un programa de tamizaje neonatal expandido en Colombia, se realizó una revisión bibliográfica sobre estos programas en diversos países, su impacto en la salud pública y el marco legal que ofrece el país para su implementación eventual.

\section{Reflexión sobre el tamizaje neonatal expandido, desde el marco de la salud pública}

Dentro de los objetivos del desarrollo sostenible (oDs), se propuso que, para 2030, debe ponerse fin a las muertes evitables de recién nacidos y menores de 5 años. Sumado al objetivo de reducir a un tercio las muertes prematuras por enfermedades no transmisibles, mediante la prevención, el tratamiento y la promoción de la salud mental y el bienestar (11). Sin embargo, no solo la mortalidad es un problema, sino también la carga mundial de 
morbilidad, pues se estima que 95 millones de niños tienen algún grado de discapacidad y, de ellos, 13 millones poseen una discapacidad grave (12).

A medida que el éxito en vacunación es mayor, se reduce la mortalidad infantil por causas infecciosas y surge una transición epidemiológica que plantea la necesidad de la prevención de las anomalías congénitas. Como muestran las estadísticas vitales del DANE, desde 2006, estas enfermedades son una importante causa de morbimortalidad en Colombia (13). En 2014, se reportaron 7589 muertes de menores de un año a nivel nacional, de las cuales 1043 fueron causadas por anomalías congénitas (14).

En este sentido, el tamizaje neonatal expandido pretende mitigar las repercusiones orgánicas y el daño neurológico, a fin de mejorar el bienestar del individuo afectado y, asimismo, el de su familia $(15,16)$. Esto lo convierte en una de las herramientas en salud pública más efectivas, pues contribuye, al mismo tiempo, a la reducción de la mortalidad infantil, de la discapacidad, del costo socioeconómico que estas enfermedades representan y del impacto sobre las familias y la comunidad (11).

\section{Revisión de la normatividad vigente en Colombia en salud infantil, enfermedades huérfanas y raras, discapacidad y tamizaje neonatal}

En Colombia, no solo la Resolución 412 de 2000 hace referencia al tamizaje neonatal para $\mathrm{HC}$, sino que, además, existen leyes, decretos, resoluciones y sentencias, las cuales ponen en evidencia el carácter obligatorio y la inmediata implementación del tamizaje neonatal expandido en el país, como derecho de todos los recién nacidos, tal como se muestra en la Tabla 1. Sin embargo, con respecto a los EIM, la normatividad no era clara y se limitaba a las recomendaciones presentadas en las Guías de Práctica Clínica de Detección de Anomalías Congénitas (2013), hasta la aparición de la Ley 1980 de 2019 (17).

Tabla 1. Marco normativo para una propuesta de tamizaje neonatal expandido (Colombia)

\section{Marco legal Concepto}

Constitución Política de Colombia 1991
El Estado tiene la función de ser garante de derechos, con mención especial a la garantía de derechos de las gestantes, niñas y niños.

Artículo 2. Son fines esenciales del Estado: servir a la comunidad, promover la prosperidad general y garantizar la efectividad de los principios, derechos y deberes consagrados en la Constitución. Artículo 13. Todas las personas nacen libres e iguales ante la Ley, recibirán la misma protección y trato de las autoridades y gozarán de los mismos derechos, libertades y oportunidades sin ninguna discriminación.

Artículo 44. Son derechos fundamentales de los niños: la vida, la integridad física, la salud y la seguridad social, la alimentación equilibrada, su nombre y nacionalidad, tener una familia y no ser separados de ella, el cuidado y amor, la educación y la cultura, la recreación y la libre expresión de su opinión. Serán protegidos contra toda forma de abandono, violencia física o moral, secuestro, venta, abuso sexual, explotación laboral o económica y trabajos riesgosos. Gozarán también de los demás derechos consagrados en la Constitución, en las leyes y en los tratados internacionales ratificados por Colombia.

Artículo 48. La seguridad social es un servicio público de carácter obligatorio que se prestará bajo la dirección, coordinación y control del Estado, en sujeción a los principios de eficiencia, universalidad y solidaridad, en los términos que establezca la Ley. Se garantiza a todos los habitantes el derecho irrenunciable a la Seguridad Social.

El Estado, con la participación de los particulares, ampliará progresivamente la cobertura de la Seguridad Social, que comprenderá la prestación de los servicios en la forma que determine la Ley. La Seguridad Social podrá ser prestada por entidades públicas o privadas, de conformidad con la Ley. Artículo 366. El bienestar general y el mejoramiento de la calidad de vida de la población son finalidades sociales del Estado. Será objetivo fundamental de su actividad la solución de las necesidades insatisfechas de salud. Para tales efectos, en los planes y presupuestos de la Nación y de las entidades territoriales, el gasto público social tendrá prioridad sobre cualquier otra asignación. 


\section{Marco legal Concepto}

Ley 100

de 1993
Normativiza el sistema de seguridad social integral, obliga a las administradoras de recursos EPS del régimen contributivo y subsidiado a la garantía de servicios, medicamentos y laboratorios incluidos en el Plan Obligatorio de Salud (POS) y a la garantía de una red de prestación de servicios suficiente y asequible a sus usuarios.
Decreto 1938

de 1993

Acuerdo 117

de 1998

(TS

Resolución

00412 de 2000

Resolución

3384 de 2000

Establecen los lineamientos del Plan de Atención Básica en Salud.

Determina los eventos de interés en salud pública.

La guía de atención del parto especifica como una actividad de obligatorio cumplimiento, la toma de muestra de sangre del cordón umbilical para la cuantificación de la hormona estimulante de la tiroides (TSH), con el fin de tamizar al recién nacido para el HC y también contiene la norma técnica para la detección temprana de las alteraciones de crecimiento y desarrollo en el menor de 10 años, que define el conjunto de actividades, procedimientos e intervenciones dirigidas a esta población, mediante las cuales se garantizan su atención periódica y sistemática, con el propósito de detectar oportunamente la enfermedad, facilitar su diagnóstico y tratamiento, reducir la duración de la enfermedad, evitar secuelas, disminuir la incapacidad y prevenir la muerte.

Define las actividades mínimas que las entidades aseguradoras, EPS y Administradora de Régimen Subsidiado (ARS), deben garantizar a sus afiliados, a partir del 1 de abril de 2001.

Define responsabilidades en cuanto a salud pública. Establece, entre otros asuntos, disposiciones para dirigir y coordinar el sector salud en su jurisdicción.

Ley 715 Artículo 44. Numeral 44.3.1. Adoptar, implementar y adaptar las políticas y planes en salud pública de de $2002 \quad$ conformidad.

Artículo 46. Las acciones de salud pública en cuanto promoción y prevención estarán a cargo de los entes territoriales, dirigidos a la población de su jurisdicción.

\begin{tabular}{|c|c|}
\hline $\begin{array}{l}\text { Ley } 10 \\
\text { de } 1990\end{array}$ & $\begin{array}{l}\text { Por la cual se reorganiza el Sistema Nacional de Salud, descentraliza la prestación de servicios en el } \\
\text { primer nivel en los municipios, donde se incluye la prevención de las enfermedades. }\end{array}$ \\
\hline $\begin{array}{l}\text { Ley } 60 \\
\text { de } 1993\end{array}$ & Los artículos 2, 3 y 4 definen competencia y recursos para la atención integral en Salud. \\
\hline $\begin{array}{l}\text { Ley } 1098 \\
\text { de } 2006\end{array}$ & $\begin{array}{l}\text { Ley de Infancia y Adolescencia. Garantiza los derechos de niños, niñas y adolescentes, en un contexto } \\
\text { de protección en todos los ámbitos del ser humano. Se refiere, específicamente, al derecho de los } \\
\text { niños y niñas a que se les brinde el acceso a los exámenes de diagnóstico, prevención, seguimiento y } \\
\text { tratamiento de los problemas congénitos y lo mismo aplica para la prevención de la discapacidad. }\end{array}$ \\
\hline $\begin{array}{l}\text { Decreto } 3518 \\
\text { de } 2006\end{array}$ & $\begin{array}{l}\text { Por el cual se crea y reglamenta el Sistema de Vigilancia en Salud Pública y se dictan otras } \\
\text { disposiciones. }\end{array}$ \\
\hline $\begin{array}{l}\text { Ley } 1122 \\
\text { de } 2007\end{array}$ & $\begin{array}{l}\text { Por el cual se hacen algunas modificaciones en el Sistema General de Seguridad Social en Salud y se } \\
\text { dictan otras disposiciones (artículos } 32 \text { y 33). }\end{array}$ \\
\hline $\begin{array}{l}\text { Ley } 4747 \\
\text { de } 2007\end{array}$ & $\begin{array}{l}\text { Obliga a la atención integral sin barreras y garantía de servicios de salud, donde los trámites } \\
\text { administrativos se hacen directamente entre las instituciones prestadoras de servicios de salud y las } \\
\text { entidades responsables del pago de servicios de salud (EPS, subsidiado y contributivo, fondos locales y } \\
\text { departamentales de salud, otros regímenes). }\end{array}$ \\
\hline $\begin{array}{l}\text { Sentencia T-760 } \\
\text { de } 2008 \text { de la Corte } \\
\text { Constitucional }\end{array}$ & Obliga a la garantía al derecho a la salud a cualquier colombiano tanto POS como no POS. \\
\hline $\begin{array}{l}\text { Ley } 1295 \\
\text { de } 2009\end{array}$ & a la atención integral en SISBEN 1, 2 y 3. \\
\hline
\end{tabular}




\begin{tabular}{|c|c|}
\hline Marco legal & Concepto \\
\hline $\begin{array}{l}\text { CONPES } 14 \\
\text { de } 2012\end{array}$ & $\begin{array}{l}\text { Distribución de los recursos del sistema general de participaciones. } \\
\text { Distribución de los recursos para la atención integral de la primera infancia, vigencia de 2012, } \\
\text { provenientes del crecimiento real de la economía superior al } 4 \% \text { en el } 2010 . \\
\text { Ajuste a la distribución de los recursos de once doceavas de } 2012 \text { de la participación de propósito } \\
\text { general. }\end{array}$ \\
\hline $\begin{array}{l}\text { CONPES } 91 \\
\text { de } 2012\end{array}$ & Metas y estrategias de Colombia para el logro de los objetivos de desarrollo del milenio, 2015. \\
\hline $\begin{array}{l}\text { CONPES } 109 \\
\text { de } 2012\end{array}$ & Política pública nacional de primera infancia: Colombia por la primera infancia. \\
\hline $\begin{array}{l}\text { CONPES } 152 \\
\text { de } 2012\end{array}$ & $\begin{array}{l}\text { Distribución de los recursos del sistema general de participaciones, distribución de los recursos para } \\
\text { la atención integral de la primera infancia, vigencia 2012, provenientes del crecimiento real de la } \\
\text { economía superior al } 4 \text { \% en } 2010 \text {. Ajuste a la distribución de los recursos de once doceavas de } 2012 \text { de } \\
\text { la participación de propósito general. }\end{array}$ \\
\hline $\begin{array}{l}\text { Decreto } 1392 \\
\text { de } 2010\end{array}$ & $\begin{array}{l}\text { Es conocida como Ley de las enfermedades huérfanas y, por medio de ella, se reconocen estas } \\
\text { enfermedades como de especial interés en salud y se adoptan normas para garantizar la protección } \\
\text { social por parte del Estado colombiano a la población afectada y a sus cuidadores. }\end{array}$ \\
\hline $\begin{array}{l}\text { Acuerdo } 029 \\
\text { de } 2011\end{array}$ & $\begin{array}{l}\text { Por el cual se sustituye el Acuerdo } 028 \text { de } 2011 \text { que define, aclara y actualiza integralmente el pos. } \\
\text { Contempla los medicamentos para el tratamiento del HC, dentro del POS (regímenes contributivo y } \\
\text { subsidiado). }\end{array}$ \\
\hline $\begin{array}{l}\text { Ley } 1438 \\
\text { de } 2011\end{array}$ & $\begin{array}{l}\text { Según señala su artículo primero, "tiene como objeto el fortalecimiento del Sistema General de } \\
\text { Seguridad Social en Salud, a través de un modelo de prestación del servicio público en salud que, } \\
\text { en el marco de la estrategia Atención Primaria en Salud, permita la acción coordinada del Estado, } \\
\text { las instituciones y la sociedad para el mejoramiento de la salud y la creación de un ambiente sano y } \\
\text { saludable, que brinde servicios de mayor calidad, incluyente y equitativo, donde el centro y objetivo de } \\
\text { todos los esfuerzos sean los residentes en el país. } \\
\text { Se incluyen disposiciones para establecer la unificación del Plan de Beneficios para todos los } \\
\text { residentes, la universalidad del aseguramiento y la garantía de portabilidad o prestación de los } \\
\text { beneficios en cualquier lugar del país, en un marco de sostenibilidad financiera. }\end{array}$ \\
\hline $\begin{array}{l}\text { Decreto } 4875 \\
\text { de } 2011\end{array}$ & $\begin{array}{l}\text { Por el cual se crea la Comisión Intersectorial para la Atención Integral de la Primera Infancia (AIPI) y la } \\
\text { Comisión Especial de Seguimiento para la Atención Integral a la Primera Infancia. }\end{array}$ \\
\hline $\begin{array}{l}\text { Plan Nacional } \\
\text { de Desarrollo } \\
2010-2014\end{array}$ & $\begin{array}{l}\text { Artículo 123. Ajuste de la oferta programática para la primera infancia. El Instituto Colombiano } \\
\text { de Bienestar Familiar (ICBF) priorizará su presupuesto en forma creciente para ser destinado a } \\
\text { la financiación de la estrategia de atención a la primera infancia. Acción Social, el Ministerio de } \\
\text { Protección Social y el Ministerio de Educación Nacional atenderán los criterios fijados en la política } \\
\text { para la atención a la primera infancia. }\end{array}$ \\
\hline $\begin{array}{l}\text { Resolución } 4505 \\
\text { de } 2012\end{array}$ & $\begin{array}{l}\text { Por la cual se establece el reporte relacionado con el registro de las actividades de protección } \\
\text { específica, detección temprana y la aplicación de las Guías de Atención Integral, para las } \\
\text { enfermedades de interés en salud pública de obligatorio cumplimiento. }\end{array}$ \\
\hline $\begin{array}{l}\text { Plan Decenal de } \\
\text { Salud Pública } \\
\text { 2012-2021 }\end{array}$ & $\begin{array}{l}\text { Es un pacto social y un mandato ciudadano, sustentado en que la salud pública es el compromiso } \\
\text { de la sociedad con la salud y es la carta de navegación para avanzar hacia el ideal de salud de los } \\
\text { colombianos (14). Se enmarca en los mandatos definidos por la Ley } 1438 \text { de } 2011 \text {, la Ley } 1122 \text { de } 2006 \text {, } \\
\text { la Ley } 715 \text { de 2001, la Ley } 152 \text { de } 1994 \text { y la Ley } 1450, \text { por medio de la cual se expide el Plan Nacional de } \\
\text { Desarrollo 2010-2014. } \\
\text { Es una expresión concreta de una política pública de Estado, que reconoce la salud como un derecho } \\
\text { humano interdependiente con otros y como dimensión central del desarrollo humano. El Plan Decenal de } \\
\text { Salud Pública se construye con enfoques que tienen en cuenta entre otros factores, los derechos de las } \\
\text { personas, las características de ciclo de vida, el género, lo étnico y los determinantes sociales, de donde } \\
\text { salen los seis ejes temáticos esenciales, entre los cuales nos compete para el tamizaje neonatal, el eje que } \\
\text { se refiere a la salud sexual y reproductiva y el que se refiere a la vida saludable y condiciones crónicas. }\end{array}$ \\
\hline
\end{tabular}




\begin{tabular}{|l|l|}
\hline Marco legal & Concepto \\
\hline $\begin{array}{l}\text { Guía de Atención } \\
\text { Integral del Recién } \\
\text { Nacido Sano 2012 }\end{array}$ & $\begin{array}{l}\text { Se considera deseable que haya una persona entrenada para hacer acompañamiento en el proceso } \\
\text { de lactancia, tamizaje del recién nacido y cuidado de la madre y el niño que pueda hacer visitas } \\
\text { domiciliarias el tercer día postparto. Al alta hospitalaria posparto se debe incluir información sobre } \\
\text { pruebas de tamizaje auditivo y metabólico. }\end{array}$ \\
\hline $\begin{array}{l}\text { Guía de Práctica } \\
\text { Clínica 2013 }\end{array}$ & $\begin{array}{l}\text { Detección de anomalías congénitas en el recién nacido de 2013: Sistema General de Seguridad } \\
\text { Social en Salud-Colombia. Para uso de profesionales de la salud 2013 Guía No. 03 que establece } \\
\text { recomendaciones para el tamizaje de eim en neonatos. }\end{array}$ \\
\hline $\begin{array}{l}\text { Ley estatutaria } \\
\text { de salud 1752 } \\
\text { de 2015 }\end{array}$ & $\begin{array}{l}\text { Por medio de la cual se regula el derecho fundamental a la salud y se dictan otras disposiciones. En } \\
\text { el artículo 11, se establece como sujetos de especial protección a los niños y niñas y se aclara que la } \\
\text { atención en salud no estará limitada por ningún tipo de restricción administrativa o económica. }\end{array}$ \\
\hline $\begin{array}{l}\text { Ley 1980 } \\
\text { de } 2019\end{array}$ & Esta Ley tiene como objeto regular y ampliar la práctica del tamizaje neonatal en Colombia. \\
\hline
\end{tabular}

Fuente: tomado con modificaciones de Valera. Propuesta de un modelo de tamizaje neonatal expandido en Colombia [maestría]. [Bogotá]: Universidad El Bosque, 2016 (17).

\section{Revisión de la literatura disponible sobre la experiencia del tamizaje neonatal expandido en Europa y Norteamérica}

En Estados Unidos, con el apoyo del director del Programa de Salud Materno Infantil del estado de Nueva York, Robert Guthrie inició un programa de tamizaje neonatal en 1962 (18). Hasta el 2000, solo estaban incluidas en el programa 10 EIM. No obstante, desde la introducción de la Ms/Ms hace casi dos décadas, los recién nacidos son tamizados para la detección de más de 30 EIM. Aun así, al no existir una política nacional sobre tamizaje, cada estado puede decidir qué enfermedades incluir dentro de sus respectivos programas, los cuales son obligatorios desde $2009(19,20)$.

En Canadá, se ofrece el tamizaje de 5-38 desórdenes metabólicos, dependiendo de cada provincia o territorio. Actualmente el tamizaje para fenilcetonuria (PKU) e HC es obligatorio en una sola provincia y, aunque se ofrece como recomendación en el cuidado del recién nacido, no es obligatorio en todo el territorio canadiense (21).

En Europa, el tamizaje neonatal para HC y PKU es obligatorio en todos los estados miembros de la Unión Europea. Varios países ya han introducido a sus programas el uso de Ms/Ms y otros, como Bulgaria, Noruega, República Checa y Rumania, están realizando estudios para el uso de este método.
Alemania fue pionero en la inclusión de la MS/MS a partir de 1998 y actualmente casi todos los estados alemanes realizan tamizaje de 10 enfermedades principales en los recién nacidos (10).

\section{Revisión de la literatura disponible sobre la experiencia de tamizaje neonatal expandido, en América Latina}

Los programas de tamizaje neonatal en América Latina no se iniciaron sino hasta mediados de la década de 1970. Su introducción fue lenta y tuvieron que pasar varios años para que fueran incluidos en los sistemas de salud y fueran constituidos programas $(18,22)$. Además, se caracterizan por la diversidad en cuanto a organización (18).

Entre 1985 y 1990, Argentina, Brasil, Chile, Colombia, Costa Rica, México, Uruguay y Venezuela iniciaron programas piloto de tamizaje neonatal o realizaban estas pruebas a demanda (22). En 2007, solo Cuba, Costa Rica, Chile y Uruguay contaban con un programa establecido, con cobertura cercana al $100 \%$ de todos los casos posibles y a cargo del Estado que, además, ofrecía tratamiento a los casos detectados (22). Le seguían Brasil, México y Argentina, con una cobertura de aproximadamente 70-80\% de los recién nacidos. Brasil, además, era el único de los tres países que tenía un programa nacional de tamizaje neonatal (22). 
Luego se encuentran Colombia, Paraguay y Venezuela, donde los programas son recientes y no se han implementado totalmente. Sin embargo, países como Nicaragua y Perú, donde los programas de pesquisa neonatal no se implementaron sino hasta 2005, en 2007 solo cubrían el 6,0\% de los recién nacidos (22).

En ese mismo año, Ecuador, Guatemala, República Dominicana, Bolivia y Panamá no tenían programas nacionales de tamizaje neonatal o realizaban mínimas actividades al respecto, a demanda y a nivel privado, con una cobertura menor al $1,0 \%$. Con todo, más grave aún es que El Salvador, Honduras y Haití no reportan actividad alguna al respecto (23).

En 2015, Cuba, Costa Rica, Uruguay y Chile seguían manejando coberturas aproximadas del 99\% de los recién nacidos. De estos países, Chile ha iniciado un proyecto piloto para incluir $25 \mathrm{EIM}$ más en su programa; Cuba añadió la fibrosis quística (FQ) en 2009, mientras Uruguay agregó al suyo PKU e hiperplasia suprarrenal congénita (HSC), en 2007, y FQ, en 2009 (23). Brasil, México y Argentina han ido añadiendo nuevas EIM a sus programas de tamizaje, mientras que Colombia y Venezuela han aumentado la cobertura.

El cambio más destacable, no obstante, ocurrió en países como Ecuador, Perú, Panamá, Nicaragua, Paraguay y Bolivia. Paraguay creó el "Programa para la Prevención de la Fibrosis Quística y el Retraso Mental producido por Hipotiroidismo Congénito y de Fenilcetonuria”, en 2003, con una cobertura que superaba el $60 \%$ para HC y PKU (23). En Panamá, el programa para tamizaje de HC y deficiencia de glucosa-6-fofato deshidrogenasa inició en 2007 e incluyeron GAL y PKU en 2008, sumado a un programa de tamizaje independiente, ofrecido por el seguro social; ambos suman en cobertura cerca del $75 \%$ de los recién nacidos. En 2005, Nicaragua inició un programa de tamizaje en la Universidad Autónoma de Nicaragua, que actualmente solo tamiza HC, con una cobertura superior al $86 \%$ de los recién nacidos (23).

Por su parte, ecuador instauró su programa de tamizaje en 2011, tamizando PKU, HC, HSC y GAL, con una cobertura del $90 \%$ de los recién nacidos. En Perú el programa se creó en 2012, cubriendo las mismas patologías que Ecuador, pero con una cobertura de apenas $20 \%$. En Bolivia, no se ha implementado como tal un programa de tamizaje, aunque en 2006 se hizo obligatorio tamizar para HC.

A pesar de los avances de la mayoría de los países, en Honduras, los programas de tamizaje se han tratado de implementar, sin éxito; mientras, en El Salvador se instauró en 2006, pero fue descontinuado debido a falta de fondos y, por último, Haití continúa sin actividades de tamizaje (23).

\section{Revisión del modelo de tamizaje neonatal de hipotiroidismo congénito en Colombia}

Desde el 2000, Colombia inició su programa de tamizaje neonatal para HC, con todos sus componentes: detección, confirmación, tratamiento, seguimiento, asesoría y vigilancia (6). A partir de entonces, la tasa de cobertura alcanza un promedio del $80 \%$ de los recién nacidos, mientras Bogotá alcanza cerca del $100 \%$. No obstante, existen diferencias interdepartamentales, manifiestas en menor cobertura en regiones con menor población, bajo desarrollo y difícil acceso geográfico, tal como se muestra en la Tabla 2 (13). 
Tabla 2. Cobertura en tamizaje neonatal de hipotiroidismo congénito por departamento, 2012

\begin{tabular}{|c|c|c|c|c|c|c|c|}
\hline Departamento & Tamizados & Rellamados & Probables & Confirmados & $\begin{array}{c}\text { Porcentaje } \\
\text { de } \\
\text { efficacia }(\%)\end{array}$ & Partos & $\begin{array}{c}\text { Porcentaje } \\
(\%)\end{array}$ \\
\hline Amazonas & 0 & 0 & 0 & 0 & 0 & 1503 & 0 \\
\hline Antioquia & 35477 & 69 & 316 & 0 & 22 & 76131 & 47 \\
\hline Arauca & 2277 & 9 & 9 & 0 & 100 & 4433 & 51 \\
\hline Atlántico & 16608 & 16 & 31 & 2 & 52 & 40022 & 41 \\
\hline Bogotá & 175163 & 720 & 2279 & 43 & 32 & 117557 & 149 \\
\hline Bolívar & 10731 & 3 & 14 & 0 & 21 & 35667 & 30 \\
\hline Boyacá & 21237 & 49 & 334 & 0 & 15 & 17150 & 124 \\
\hline Caldas & 10478 & 65 & 108 & 2 & 60 & 10370 & 101 \\
\hline Caquetá & 17717 & 31 & 155 & 2 & 20 & 7739 & 229 \\
\hline Casanare & 4506 & 6 & 27 & 1 & 22 & 6733 & 67 \\
\hline Cauca & 4338 & 33 & 60 & 2 & 55 & 15812 & 27 \\
\hline Cesar & 4713 & 3 & 23 & 0 & 13 & 19909 & 24 \\
\hline Chocó & 735 & 0 & 0 & 0 & 0 & 3458 & 21 \\
\hline Córdoba & 10372 & 37 & 72 & 0 & 51 & 28918 & 36 \\
\hline Cundinamarca & 123 & 1 & 0 & 0 & 0 & 23124 & 1 \\
\hline Guainía & 0 & 0 & 0 & 0 & 0 & 482 & 0 \\
\hline Guajira & 0 & 0 & 0 & 0 & 0 & 12980 & 0 \\
\hline Guaviare & 0 & 0 & 0 & 0 & 0 & 1166 & 0 \\
\hline Huila & 28618 & 239 & 350 & 7 & 68 & 21125 & 135 \\
\hline Magdalena & 514 & 0 & 1 & 0 & 0 & 23218 & 2 \\
\hline Meta & 21407 & 165 & 283 & 9 & 58 & 16351 & 131 \\
\hline Nariño & 12944 & 91 & 190 & 4 & 48 & 17713 & 73 \\
\hline $\begin{array}{l}\text { Norte de } \\
\text { Santander }\end{array}$ & 7860 & 30 & 84 & 1 & 36 & 20418 & 38 \\
\hline Putumayo & 4734 & 39 & 63 & 5 & 62 & 3991 & 119 \\
\hline Quindío & 242 & 0 & 4 & 0 & 0 & 6210 & 4 \\
\hline Risaralda & 4216 & 3 & 32 & 0 & 9 & 12157 & 35 \\
\hline San Andrés & 1534 & 7 & 11 & 0 & 64 & 805 & 191 \\
\hline Santander & 23292 & 10 & 60 & 1 & 17 & 31444 & 74 \\
\hline Sucre & 3471 & 7 & 20 & 3 & 35 & 15291 & 23 \\
\hline Tolima & 18941 & 61 & 130 & 4 & 47 & 15291 & 103 \\
\hline Valle & 89619 & 227 & 604 & 15 & 38 & 56770 & 158 \\
\hline Vaupés & 123 & 1 & 2 & 0 & 50 & 596 & 21 \\
\hline Vichada & 305 & 0 & 1 & 1 & 0 & 565 & 54 \\
\hline Total & 532172 & 1921 & 5263 & 102 & 0 & 668197 & 80 \\
\hline
\end{tabular}

Fuente: Valera. Propuesta de un modelo de tamizaje neonatal expandido en Colombia [maestría]. [Bogotá]: Universidad El Bosque, 2016 (17). 


\section{Revisión de la literatura sobre carga económica y relación costo-beneficio del programa de tamizaje neonatal expandido con espectrometría de masas (tándem)}

Como se mencionó, algunos países latinoamericanos, como Costa Rica y Uruguay, tienen en marcha programas de tamizaje neonatal universales, mediante el uso de la espectrometría de masas en tándem (MS/Ms). Este método permite detectar diversos trastornos metabólicos asociados con el metabolismo de aminoácidos, ácidos grasos y ácidos orgánicos, a través de una sola muestra de sangre seca en papel filtro (24).

Rosselli y su equipo evidenciaron la factibilidad y costo-efectividad de realizar un programa de tamizaje similar en Colombia. Su análisis de costos se centró en el tamizaje mediante Ms/Ms, teniendo en cuenta los costos de las pruebas confirmatorias y el costo por caso detectado de alguna de las siguientes condiciones: fenilcetonuria, galactosemia, deficiencia de biotinidasa, hiperplasia suprarrenal congénita, deficiencia de acil-CoA-deshidrogenasa de cadena media y acidemias orgánicas (24).

Los autores sugieren que, en el primer año, se trabajaría al $50 \%$ de la capacidad; al $80 \%$, el segundo año y al $90 \%$ el resto del tiempo, con un costo mensual de 700000 pesos colombianos de arriendo mensual y 6,5 millones de pesos más prestaciones para una nómina de tres personas que usan el equipo (microbiólogo, asistente de laboratorio y coordinador). El costo por prueba sería de 19281 pesos, más $\$ 939$ del papel filtro, más 1400 pesos de costos de envío, aproximadamente (24).

En el caso del primer espectrómetro cotizado, el costo de cada prueba en el primer año sería de
30915 pesos y el valor de la inversión se recuperaría el cuarto año. Así, se estarían tamizando alrededor de 40000 recién nacidos y el costo por caso detectado sería de 186360812 de pesos. Con el segundo espectrómetro, el panorama no sería muy diferente, excepto por el costo del equipo, que sería más elevado. En este caso, el costo por prueba sería de 33499 pesos; la inversión también se recuperaría a los cuatro años; la cobertura sería similar y el costo por caso detectado sería de 306850662 de pesos (24).

Los autores también proponen el uso de varios equipos de MS/MS, distribuidos en diferentes regiones del país, con lo cual se tamizarían 518400 recién nacidos al año y el costo de la prueba reduciría en $25 \%$. Sin embargo, deben considerarse factores adicionales como el acceso geográfico, la sensibilización al personal de salud y la comunidad general; así como la infraestructura y personal capacitado necesarios (24).

\section{Presentación de una propuesta para implementar un modelo de tamizaje neonatal expandido, en Colombia}

Aquí se propone la conformación de un Centro Nacional de Tamizaje Neonatal, encargado de planear, organizar, direccionar, coordinar, integrar, evaluar, retroalimentar y ajustar el programa de tamizaje (Figura 1). Las empresas administradoras de los planes de beneficios (ЕАРB) tendrán a su cargo todo lo correspondiente a los trámites administrativos que requiera el proceso de atención. Asimismo, deberán establecerse laboratorios especializados que realicen las pruebas de confirmación o complementarias que se requieran para el diagnóstico adecuado de cada enfermedad detectada, como se muestra en la Figura 1 (17). 


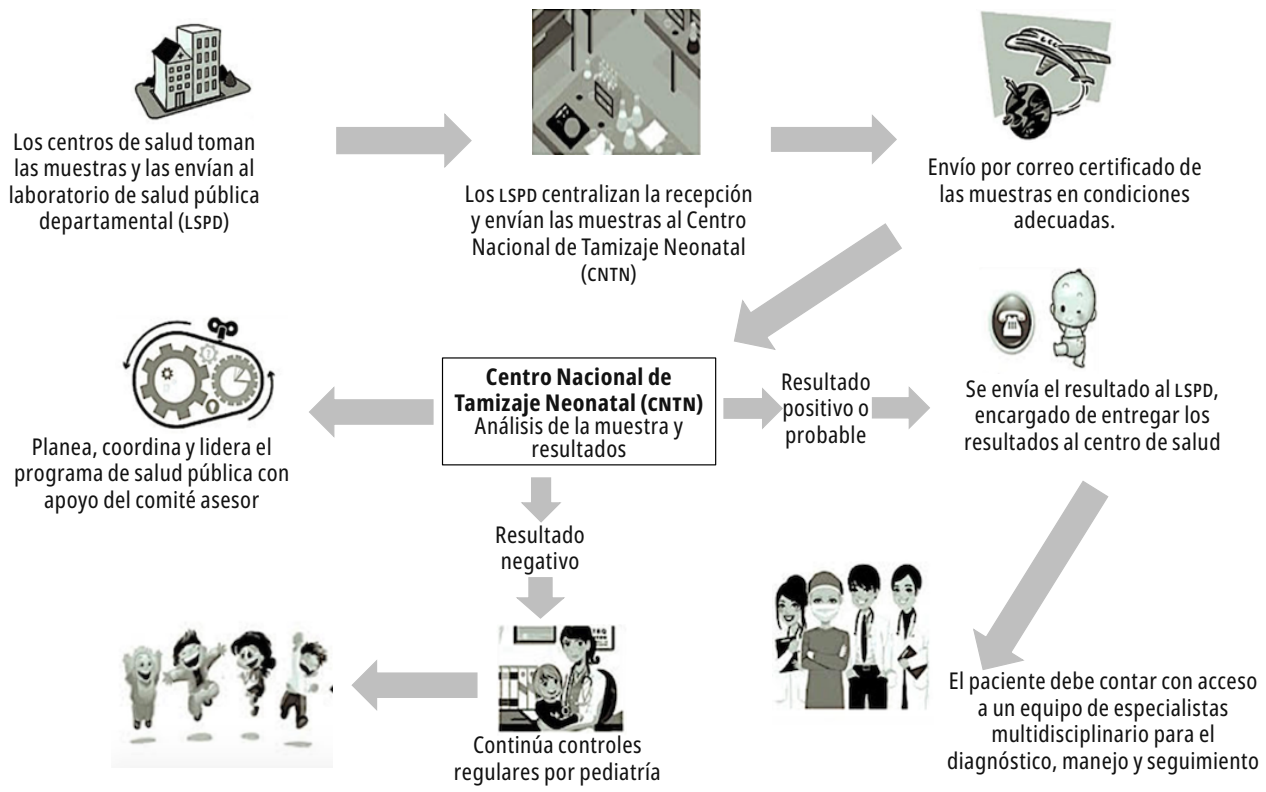

Figura 1. Flujograma del modelo de tamizaje neonatal expandido en Colombia.

Fuente: imagen tomada y modificada de Valera. Propuesta de un modelo de tamizaje neonatal expandido en Colombia [maestría]. [Bogotá]: Universidad El Bosque, 2016 (17).

Deberán definirse no solo las instituciones prestadoras de servicios de salud (IPs), sino también las clínicas o consultorios que cuenten con la infraestructura y el recurso humano necesario para el manejo de estas enfermedades, así como la posibilidad de ofrecer asesoría genética adecuada a las familias que lo requieran (17).

También, debe asegurarse el envío de las muestras hacia los laboratorios. Para ello, debe existir un servicio de correo certificado que cumpla con las normas de calidad y garantice el transporte oportuno de las muestras. El costo del tamizaje neonatal estaría financiado por las ЕАРв y la población más pobre no afiliada a estas empresas debería estar bajo la responsabilidad del Estado. Por lo cual deberá realizarse una articulación entre estas y las IPs, públicas o privadas, y establecer una red de prestadores de servicios expertos en el manejo de las enfermedades raras, entre estas, los EIM (17).

Las patologías que se sugiere incluir dentro del tamizaje neonatal ampliado en Colombia deberán ser analizadas y determinadas por un comité asesor, conformado por expertos de la sociedad médico-científica, teniendo en cuenta los criterios que se establecen en los Principles of early disease detection, que se declaran universales para el tamizaje de una enfermedad (25). En 2008, estos criterios fueron revisados por la oms y modificados, de modo que se les dio un mejor enfoque con respecto a los objetivos de desarrollo del milenio, priorizando la prevención y manteniendo un equilibrio costo-beneficio [Figura 2] (26).

\begin{tabular}{|c|c|}
\hline \multicolumn{2}{|c|}{ Criterios de tamizaje neonatal 2008} \\
\hline $\begin{array}{l}\text { El programa de tamizaje debe surgir } \\
\text { como respuesta a una necesidad } \\
\text { reconocida e } \\
\text { importante en salud pública. }\end{array}$ & $\begin{array}{l}\text { Al iniciar la implementación del } \\
\text { programa, los objetivos deben estar } \\
\text { claramente establecidos. }\end{array}$ \\
\hline $\begin{array}{l}\text { La población a la cual se va a dar } \\
\text { acceso al tamizaje debe estar } \\
\text { definida. }\end{array}$ & $\begin{array}{c}\text { Debe haber evidencia científica de la } \\
\text { eficacia del programa de } \\
\text { tamizaje, como sustento para su } \\
\text { implementación. }\end{array}$ \\
\hline $\begin{array}{l}\text { El programa debe integrar la } \\
\text { educación al personal en cuanto al } \\
\text { proceso analítico de las muestras, } \\
\text { los servicios clínicos ofrecidos al } \\
\text { paciente y la gerencia misma del } \\
\text { programa. } \\
\end{array}$ & $\begin{array}{c}\text { Debe existir garantía de calidad del } \\
\text { programa, aumentando la seguridad } \\
\text { de las pruebas con los mecanismos } \\
\text { adecuados para reducir, cuanto sea } \\
\text { posible, los riesgos potenciales del } \\
\text { tamizaje. } \\
\end{array}$ \\
\hline $\begin{array}{l}\text { El programa debe asegurar el } \\
\text { consentimiento informado a los } \\
\text { padres, la confidencialidad } \\
\text { y el respeto a la autonomía que } \\
\text { merece el individuo afectado. }\end{array}$ & $\begin{array}{l}\text { El programa debe promover la } \\
\text { equidad y el acceso a la prueba para } \\
\text { toda la población objetivo. }\end{array}$ \\
\hline $\begin{array}{l}\text { La evaluación del programa debe } \\
\text { planearse desde el principio. }\end{array}$ & $\begin{array}{c}\text { Los beneficios totales del tamizaje } \\
\text { deben compensar las molestias y } \\
\text { daños causados a los que padecen } \\
\text { las enfermedades incluidas en el } \\
\text { programa. }\end{array}$ \\
\hline
\end{tabular}

Figura 2. Criterios de tamizaje neonatal. 
Adicionalmente, este comité permitiría evaluar y plantear opciones de mejoría del programa. El comité debe ser independiente e interdisciplinario; y sus miembros deben hacer parte de él con una periodicidad especificada en la conformación del comité, con el objetivo de garantizar participación plural, darle transparencia y visión más amplia. El comité podría hacer propuestas de manera activa o evaluar las propuestas de las asociaciones de pacientes y la comunidad. El comité podría, además, presentar al Ministerio de Salud y Protección Social un concepto escrito completo, claro, preciso y detallado sobre el tema en evaluación. Finalmente, el comité sería una instancia asesora para el Ministerio de Salud y Protección Social (Figura 3).

Para el desarrollo adecuado y el éxito de este programa se hace necesaria la educación a la comunidad en general y al personal de salud, para que, además, estos profesionales estén en la capacidad de solicitar las pruebas y promover su utilización dentro de las comunidades donde realizan su trabajo.

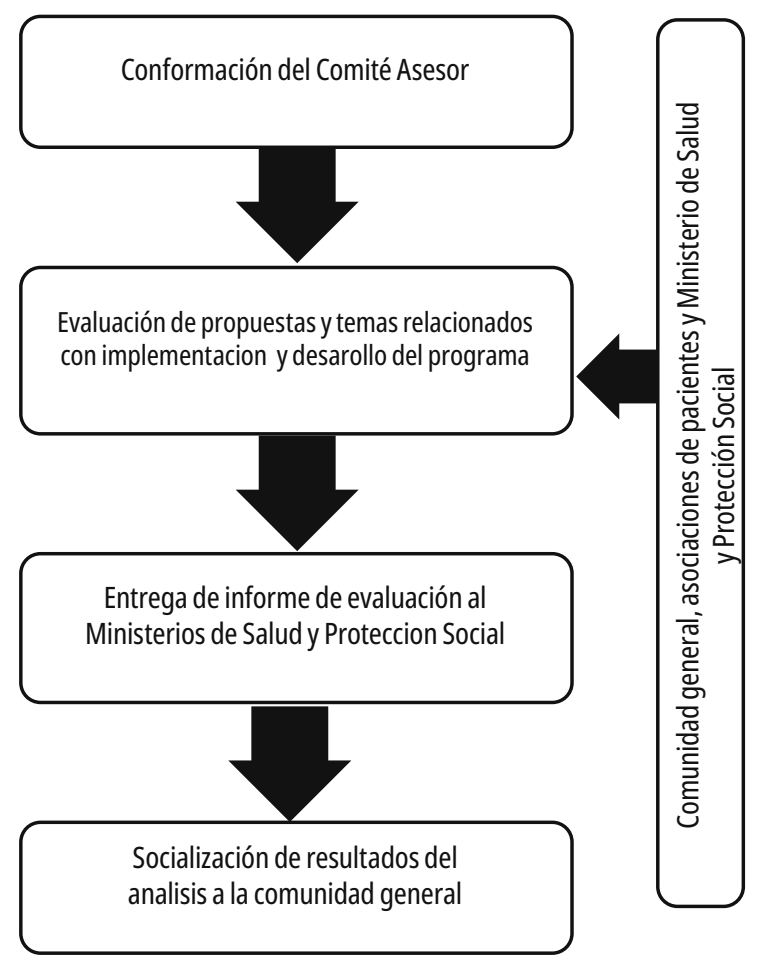

Figura 3. Evaluación de propuestas y temas relacionados con implementación y desarrollo del programa de tamizaje neonatal ampliado.

\section{Recomendaciones y conclusiones}

A pesar de que muchos países, tanto europeos como americanos, han implementado dentro de sus políticas de salud pública el tamizaje de varios EIM, aún no hay homogeneidad en los programas en cuanto a la estrategia de muestreo, número de EIM tamizados o metas de cobertura. Sin embargo, si hay un consenso universal en la necesidad de implementar programas nacionales, respaldados con normas específicas y concretas de obligatoriedad para su implementación.

Para lograr los objetivos de esta propuesta, se hace necesaria la articulación de los sectores públicos y privados, de la comunidad médico-científica, asociaciones de pacientes y comunidad general. Adicionalmente, es de gran importancia el establecimiento de una ley o acto administrativo que normatice este programa en todo el país (17). Lo cual se logró con la sanción de la Ley 1980 de 2019, que regula el tamizaje neonatal como práctica de salud pública.

En la actualidad, Colombia tiene normatividad de base que permitiría la implementación de un Programa de Tamizaje Neonatal Expandido, capaz de cubrir casi la totalidad de recién nacidos a lo largo del país, de manera obligatoria. En este sentido, deben evaluarse propuestas de implementación como las de Roselli y sus colaboradores y plantear opciones adicionales. Finalmente, la idea es implementar un programa nacional de tamizaje neonatal ampliado liderado por el Estado, con apoyo y participación constante de la academia, los profesionales de salud, las asociaciones de pacientes y también la industria farmacéutica.

\section{Referencias}

1. Rosselli D, Rueda J. Enfermedades raras, huérfanas y olvidadas [Internet]. [Actualizado en 2011; citado el 10 de septiembrede2016].Disponibleenhttp://www.academia.edu/ 5271337/estudio_enfermedades_raras_huérfanas_y_olvidadas

2. Couce ML, Fernández JR, Fraga JM. Enfermedades congénitas del metabolismo en el periodo neonatal. En: Asociación Española de Pediatría, Sociedad Española de Neonatología, editores. Protocolos de neonatología (en revisión) [Internet]. 2da edición. Madrid: Asociación Española de pediatría; 2008 [citado el 18 de diciembre de 2019]. Pp. 434-42. Disponible en https:// www.aeped.es/sites/default/files/documentos/45.pdf 
3. Koletzko B. Pediatric Nutrition in Practice. World Review of Nutrition and Dietetics. 2da edición. Munich: Karger; 2015.

4. Guzmán-Suárez ов. Certificación de discapacidad como herramienta para la accesibilidad a derechos e inclusión social. Rev. Salud pública. 2013; 15(1):149-57.

5. Pourfarzam M, Zadhoush F. Newborn screening for inherited metabolic disorders; news and views. J Res Med Sci. 2013; 18(9):801-8.

6. Colombia. Ministerio de Salud. Resolución número 412 de 2000. Bogotá: Ministerio de Salud; 2000.

7. Colombia. Ministerio de Salud y Protección Social. Guía de práctica clínica: Detección de anomalías congénitas en el recién nacido. Guía No. 03. Para uso de profesionales de la salud. Bogotá: Ministerio de Salud y Protección Social; 2013.

8. Bermúdez A, Valera D, Robayo D, Ascencio A, Ching R. Desarrollo de la tamización neonatal en Colombia: espectrometría de masas en tándem. Pediatría. 2015; 48:47-54.

9. Waisbren sE. Expanded newborn screening: Information and resources for the family physician. Am Fam Physician. 2008; 77:987-94.

10. Campos Hernández D. Tamizaje neonatal por espectrometría de masas en tándem: actualización. Rev Panam Salud Publica. 2010; 27:309-18.

11. Naciones Unidas. Objetivos del Desarrollo Sostenible. Diecisiete objetivos para transformar nuestro mundo [Internet]. Estados Unidos: Nueva York [NY]. 2015 [citado el 14 de septiembre de 2016]. Disponible en http://www.un.org/sustainabledevelopment/es/objetivos-de-desarrollo-sostenible/

12. Organización Mundial de la Salud (oMs). Resumen informe mundial sobre la discapacidad. Ginebra: oms; 2011.

13. Colombia. Dirección de Redes en Salud Pública. Tamizaje neonatal vigilancia por laboratorio actualización de recomendaciones técnicas y operativas para el laboratorio [Internet]. Bogotá: Instituto Nacional de Salud; 2014 [citado el 2 de octubre de 2016]. Disponible en http://studylib.es/ doc/7896139/isbn-978-958-13-0169-0--instituto-nacional-de-salud

14. Departamento Administrativo Nacional de Estadística (DANE).Defunciones no fetales, defunciones menores de un año. Por causas de defunción [Internet]. Colombia: Bogotá;2014 [citado el 15 de enero de 2016]. Disponible en http:// www.dane.gov.co/index.php/estadisticas-por-tema/ salud/nacimientos-y-defunciones/defunciones-no-fetales

15. Einöder M, Atienza G. Efectividad clínica del cribado neonatal de errores congénitos del metabolismo mediante espectrometría de masas en tándem. Parte I. Madrid: Red Española de Agencias de Evaluación de
Tecnologías y Prestaciones del sns; Agencia de Evaluación de Tecnologías Sanitarias de Galicia; Informes de Evaluación de Tecnologías Sanitarias. 2013. Disponible en https://www.sergas.es/Docs/Avalia-t/avalia_ t201203CribadoMetabolopatias.pdf

16. Viloria Y. ¿Qué es el tamiz neonatal ampliado? [Internet]. México; 2011 [citado el 18 de septiembre de 2016]. Disponible en https://sumedico.com/qu-es-el-tamiz-neonatal-ampliado/

17. Valera D. Propuesta de un modelo de tamizaje neonatal expandido en Colombia [maestría]. [Bogotá]: Universidad El Bosque; 2016.

18. Uruguay. Centro de Estudios Seguridad Social, Salud y Administración. Pesquisa neonatal: lo que puede prevenir una gota de sangre. Montevideo: Instituto de Seguridad Social; 2010.

19. Centers for disease control and prevention (CDC). Newborn screening laboratory bulletin [Internet]. Atlanta [GA]; 2008 [citado el 5 de febrero de 2017]. Disponible en https://www.cdc.gov/ nbslabbulletin/bulletin.html

20. Dietzen DJ, Rinaldo P, Whitley RJ, Rhead wJ, Hannon wH et al. National Academy of Clinical Biochemistry Laboratory Medicine Practice Guidelines: Follow-Up testing for metabolic disease identified by expanded newborn screening using tandem mass spectrometry. Executive summary. Clin Chem. 2009; 55:1615-26.

21. Hayeems RZ, Miller FA, Bombard Y, Avard D, Carroll J, Wilson B, Little J et al. Expectations and values about expanded newborn screening: a public engagement study. Health Expect. 2015; 18(3):419-29.

22. Borrajo GJC. Newborn screening in Latin America at the beginning of the $21^{\text {st }}$ century. J Inherit Metab Dis. 2007; 30:466-81.

23. Therrell BL, David Padilla C, Loeber JG, Kneisser I, Saadallah A, Borrajo GJC et al. Current status of newborn screening worldwide: 2015. Semin Perinatol. 2015; 39:171-87.

24. Rosselli D, Rueda J, Ruiz-Patiño A. Análisis de costos de la tamización neonatal universal mediante espectrometría de masas en tándem para errores innatos del metabolismo en Colombia. Pediatría. 2014; 47:68-73.

25. Wilson JM, Jungner G. Principles and practice of screening for disease. wHo Public Health Papers. No 34 [Internet]: Ginebra; 1968 [citado el 20 de octubre de 2016]. Disponible en http://apps. who.int/iris/bitstream/10665/37650/17/wHO_PHP_34.pdf

26. Andermann A, Blancquaert I, Beauchamp S, Déry V. Revisiting Wilson and Jungner in the genomic age: a review of screening criteria over the past 40 years. Bulletin of the World Health Organization. 2008; 86(4):241-320. 